\title{
Optical bistable devices as sensing elements
}

\author{
A.P. Gonzalez-Marcos ${ }^{1}$, A. Hurtado ${ }^{2}$, J.A. Martin-Pereda ${ }^{3}$ \\ E.T.S. Ingenieros de Telecomunicación. Universidad Politécnica de Madrid \\ Ciudad Universitaria. 28040 Madrid. Spain
}

\begin{abstract}
The nonlinear optical properties of many materials and devices have been the main object of research as potential candidates for sensing in different places. Just one of these properties has been, in most of the cases, the basis for the sensing operation. As a consequence, just one parameter can be detected. In this paper, although just one property will be employed too, we will show the possibility to sense different parameters with just one type of sensor. The way adopted in this work is the use of the optical bistability obtained from different photonic structures. Because this optical bistability has a strong dependence on many different parameters the possibility to sense different inputs appears. In our case, we will report the use of some non-linear optical devices, mainly Semiconductor Optical Amplifiers, as sensing elements. Because their outputs depend on many parameters, as the incident light wavelength, polarization, intensity and direction, applied voltage and feedback characteristics, they can be employed to detect, at the same time, different type of signals. This is because the way these different signals affect to the sensor response is very different too and appears under a different set of characteristics.
\end{abstract}

Keywords: optical sensor, DFB laser diode sensor, FP laser diode sensor

\section{INTRODUCTION}

The first studies reported about optical bistability in laser diode come from the sixties ${ }^{1}$. Main works were done during eighties, most relevant are recompiled on [2] mainly in passive optical bistability; following some of the previous ${ }^{2-7}$ works on optical semiconductor oscillators and amplifiers. In the last years it has been an intensive research topic due to the huge potential applications of these devices in different fields of technology, such as optical computing and optical communications and for the practical advantages of laser amplifiers: the presence of gain, fast response, low optical power requirements to achieve bistability ${ }^{8-9}$ etc. As a matter of fact, in optical computing, the nonlinear behavior exhibited by the laser amplifier leads to the possibility of using this kind of devices as basic components in the developing of logic gates. In the other hand, in optical communications these devices could be employed in optical switching applications, optical signal regeneration and optical head packet processing, in addition the usual use in long range links.

The more intensively studied mode of operation of laser diode amplifiers has been the transmission mode of operation, which is characterized by the appearance of anticlockwise (or S-Shaped) bistable loops. More recently the reflective mode of operation has been also studied, having been demonstrated the existence of new forms of bistability apart from the anticlockwise bistable loop, such as the X-shaped and the clockwise bistable loop. This new shapes of bistability suggest new possibilities for practical applications of this kind of devices. Anyway, the optical bistability either in reflection or in transmission, is very strongly dependent on the bias current applied to the laser amplifier, and also on the frequency detuning existing between the external optical signal and the resonant frequency mode of the laser, showing that very small changes on this parameter causes very strong variations in the input-output optical power relation of the laser amplifier.

The main objective of this paper is to present some possible applications of this strong frequency dependence of the optical bistability in laser amplifiers, in the development of structures, capable to detect very small shifts on the

\footnotetext{
1 agonmar@tfo.upm.es

2 ahurtado@tfo.upm.es

3 jamp@tfo.upm.es
} 
wavelength of a particular optical signal. This wavelength shifts could eventually cause failures in optical communication systems that are very sensitive to variations on the wavelength, such as the DWDM (Dense Wavelength Division Multiplexing) optical communication networks, where the wavelength spacing between channels is very small.

\section{SEMICONDUCTOR LASER AS SHIFT WAVELENGTH SENSOR}

The first proposed structure for the wavelength shift sensor based in laser amplifiers is shown schematically in fig. 1 . Two lasers are used, the first one being operated in reflection and the second one in transmission, where the input signal of the second laser is directly the output signal of the first one. This structure, whose transfer function has been analyzed theoretically and was based initially on the transfer function obtained from a Self Electrooptic Effects Device SEED ${ }^{10}$, has been previously proposed by the authors for the construction of optical logic cells in the $1550 \mathrm{~nm}$ wavelength range ${ }^{11}$. In this previous application, a constant and unique frequency was used for the external optical signal entering to the device. Nevertheless, as this structure is based on semiconductor laser amplifiers, which exhibit a strong dependence on the frequency detuning, it is expected that the input-output power response of the complete structure will also vary when the frequency of the external signal is modified.

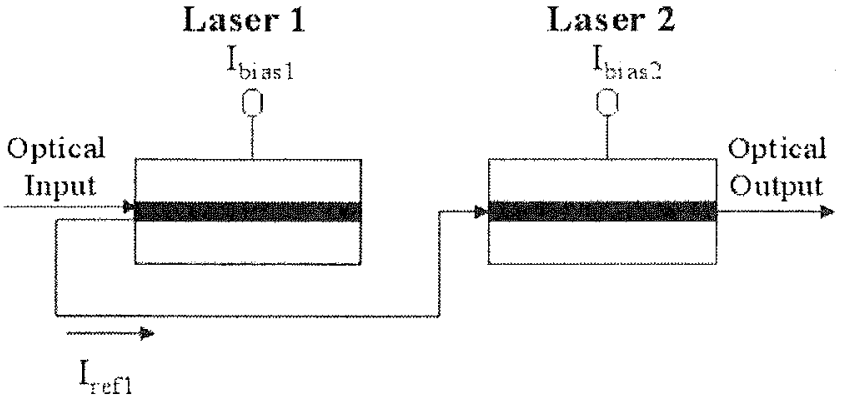

Figure 1.- Structure of the semiconductor laser wavelength shift sensor.

This structure has been studied using different types of semiconductor laser. In Fig. 2
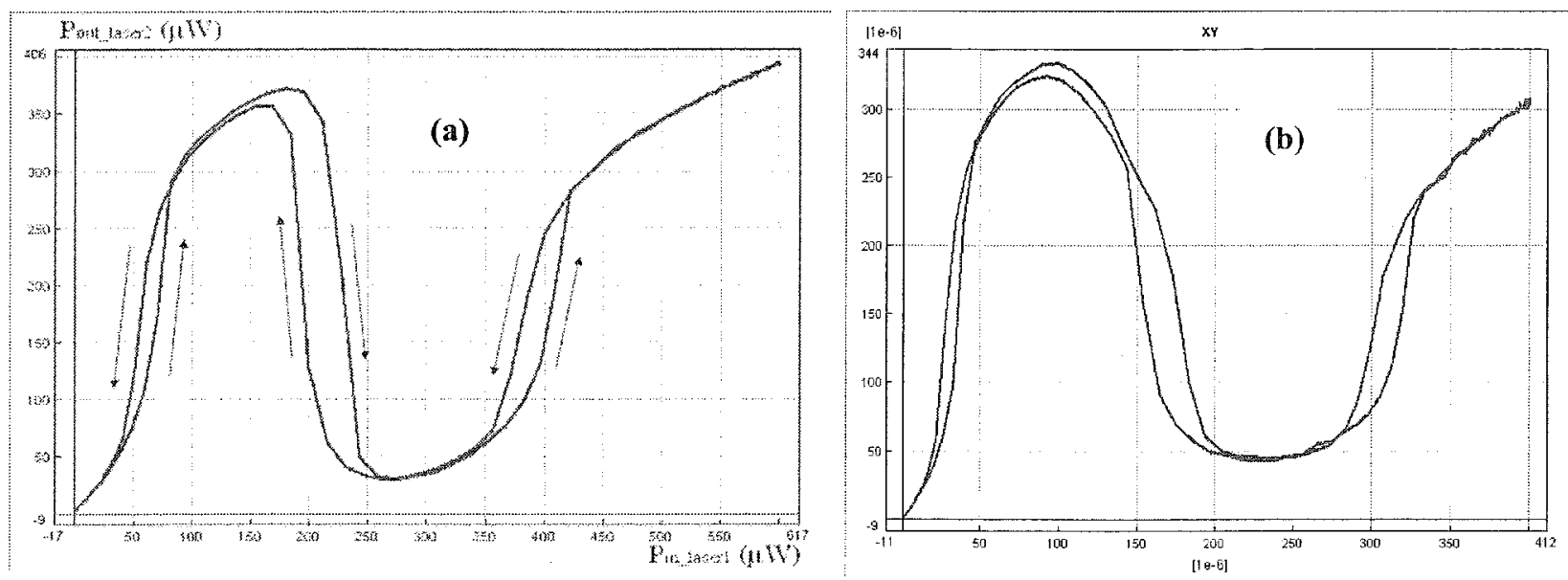

Figure 2.- Optical transfer function, $\left[\mathrm{P}_{\text {in }}(\mu \mathrm{W}), \mathrm{P}_{\text {out }}(\mu \mathrm{W})\right]$ of the structure in Fig.1.: (a) with FP Fabry-Perot diode laser; and (b)with DFB Distributed FeedBack laser diode.

A verification of the frequency dependence has been done; the optical input-output relation show on figure 2 is different depending on the external signal frequency. The first study of a wavelength sensor done with FP laser diode was reported by us on [12], on that publication it was presented the different transfer function for at least 4 values of the frequency of the optical signal entering to the device, with a frequency difference among them of only a few GHz. An increase in frequency makes the transfer function of the device to suffer a 'compression' effect, as the power requirements to achieve bistability for the lasers are lowered due to the reduction of the relative frequency detuning. In the opposite side an external signal frequency decrease, leads to a higher frequency detuning, and as the power needed 
for bistability is increased, the transfer function suffers a 'relaxation' effect. As a consequence of this behavior, if a constant optical power signal is injected to this device, the amount of optical power obtained at the output of the device will depend on the frequency of the signal.

\section{Wavelength shift sensor}

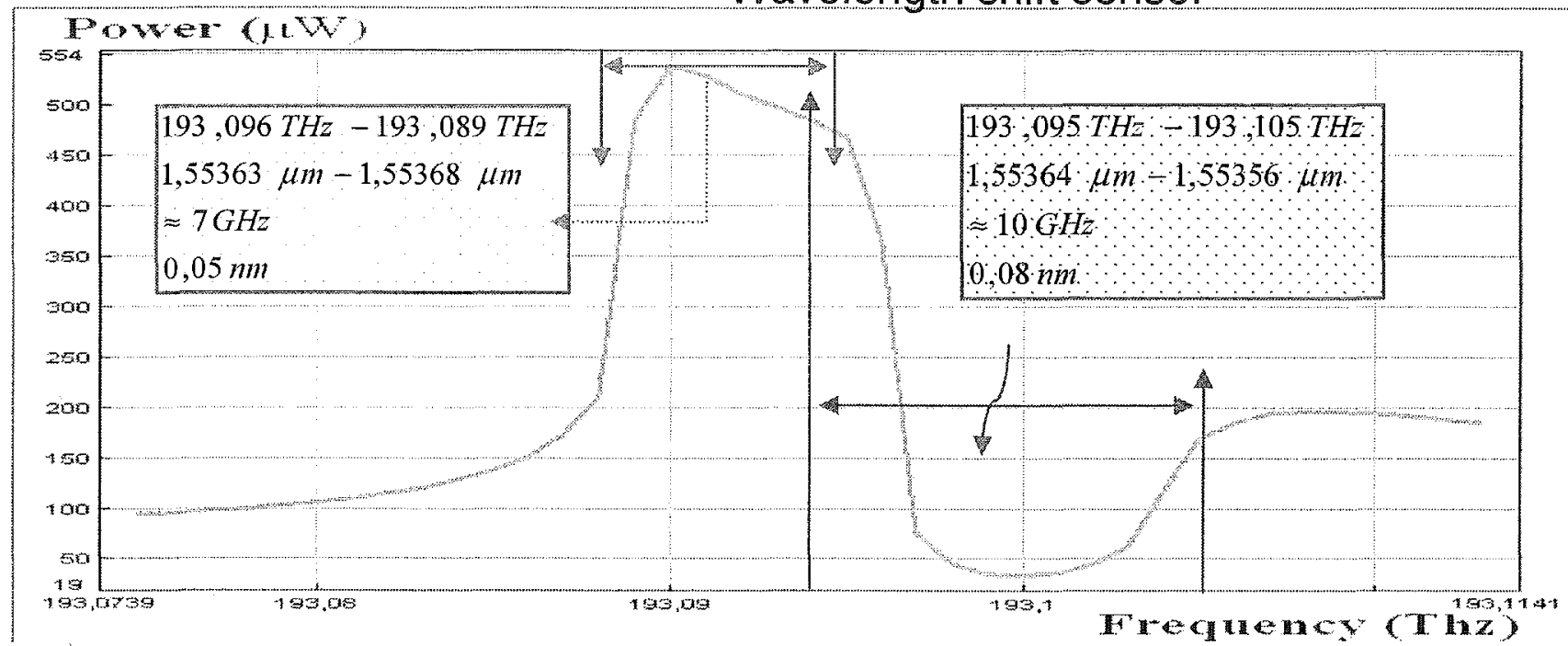

Figure 3.- Optical output power as a function of the frequency. $0.25 \mathrm{~mW}$ input signal amplitude. Results for FP laser diode structure on Fig. 1.

Fig. 3 shows the output power of the device when a $0.25 \mathrm{~mW}$ constant power optical signal is injected into it, as a

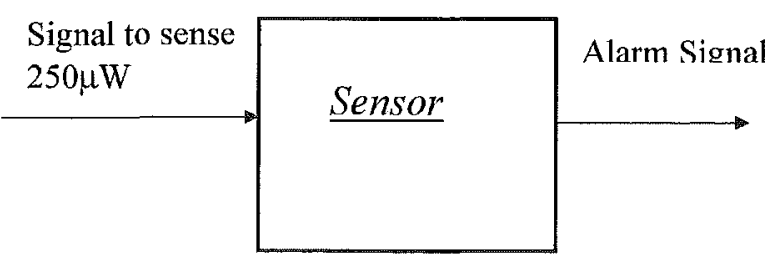

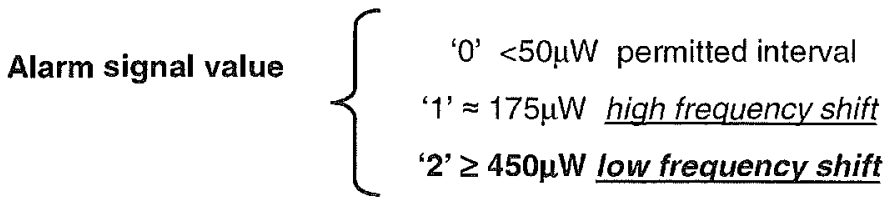

Alarm signal value $\left\{\begin{array}{l}\text { '0’ }>450 \mu W \text { permitted interval } \\ ' 1 \text { ' }<450 \mu W \text { frequency shift }\end{array}\right.$

Figure 4.- Summarize of input and output power for frequency detuning. Alarm characterization. Block Sensor is Fig. 1 with FP laser diode. function of its frequency, in a $40 \mathrm{Ghz}$ wide interval around $193.1 \mathrm{Thz}(1552.52 \mathrm{~nm})$. The graphical representation obtained for that frequency interval shows a 'mountainvalley' like shape, where different zones of frequency supply different values of constant output power. The main conclusion given by this representation is that when a constant input power signal is injected into the device, adjacent narrow intervals of the signal frequency respond with different constant output power values, separated by abruptly change zones. This behavior suggest the application of this kinds of devices as detectors of possible wavelength shifts of the emission frequency of the lasers that conform the individual multiplexed channels in Dense Wavelength Division Multiplexed (DWDM) optical telecommunication networks, by sending an optical alarm signal when a shift in wavelength is produced, and also by the amplitude of this alarm signal the direction of the shift in wavelength could be determined.

Fabry- Perot laser diodes are original semiconductor laser structures where most of the bistability studies were done at the beginning. The advantage of DFB laser diode is clear on optical communications, important bistability reports on transmission and reflection configuration, both needed for our sensor device can be found on [13-14]. We 
have also analysis how respond a DFB laser on our basic structure, used by as for optical computing ${ }^{15}$ and sensors. A comparison between noise present in a DFB and FP laser structure can be found on [16].

An study of optical input-output characteristic on sensor with DFB laser diode, as has been done previously with the FP structure, we find again the frequency dependence and results are summarized on Fig. 5 and 6.

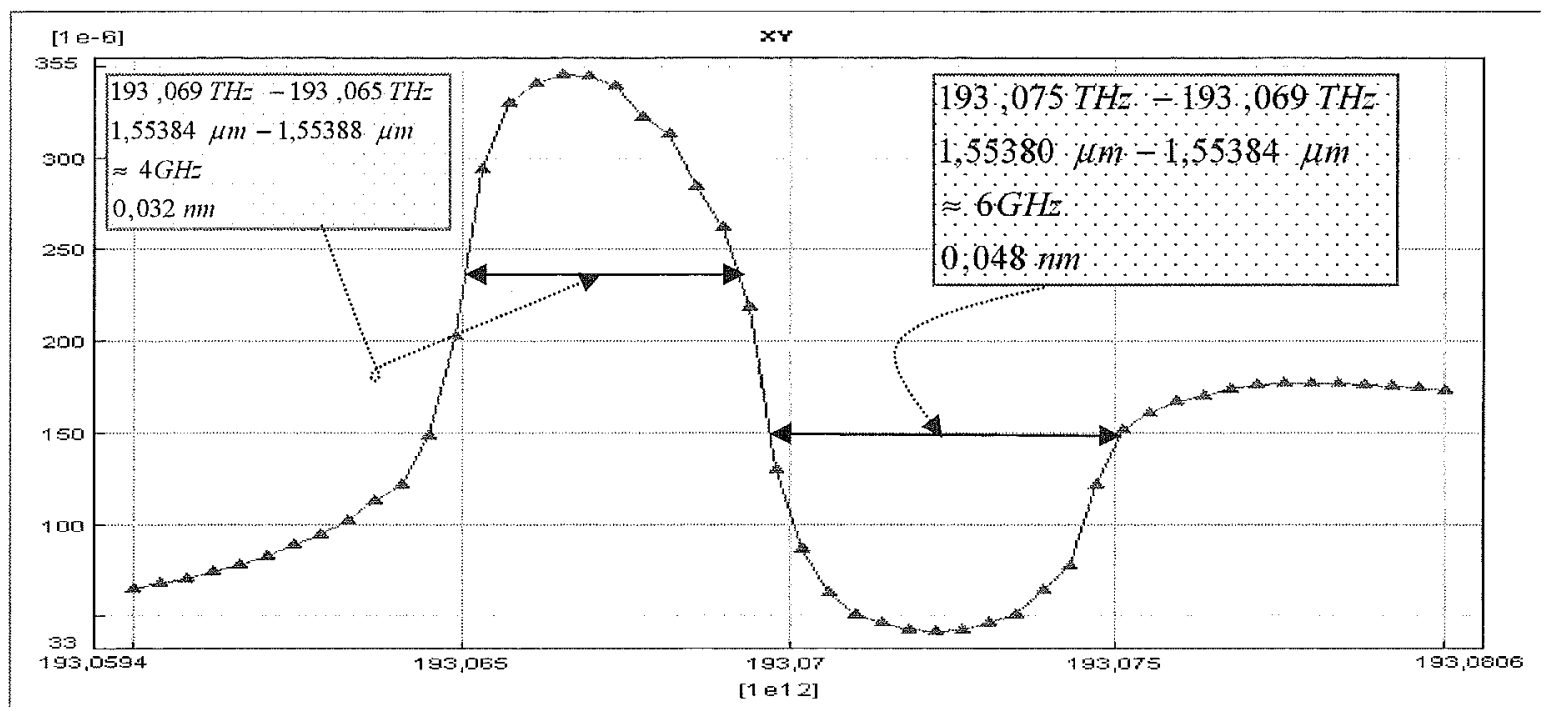

Figure 5.- Optical output power as a function of the frequency. $0.25 \mathrm{~mW}$ input signal amplitude. Results of DFB laser diode structure on Fig. 1.

Fig. 5 shows the output power of the device when a $0.25 \mathrm{~mW}$ constant power optical signal is injected into it, as a function of its frequency, in a $20 \mathrm{Ghz}$ wide interval around 193.07Thz $(1553.84 \mathrm{~nm})$. This is the first time this result are

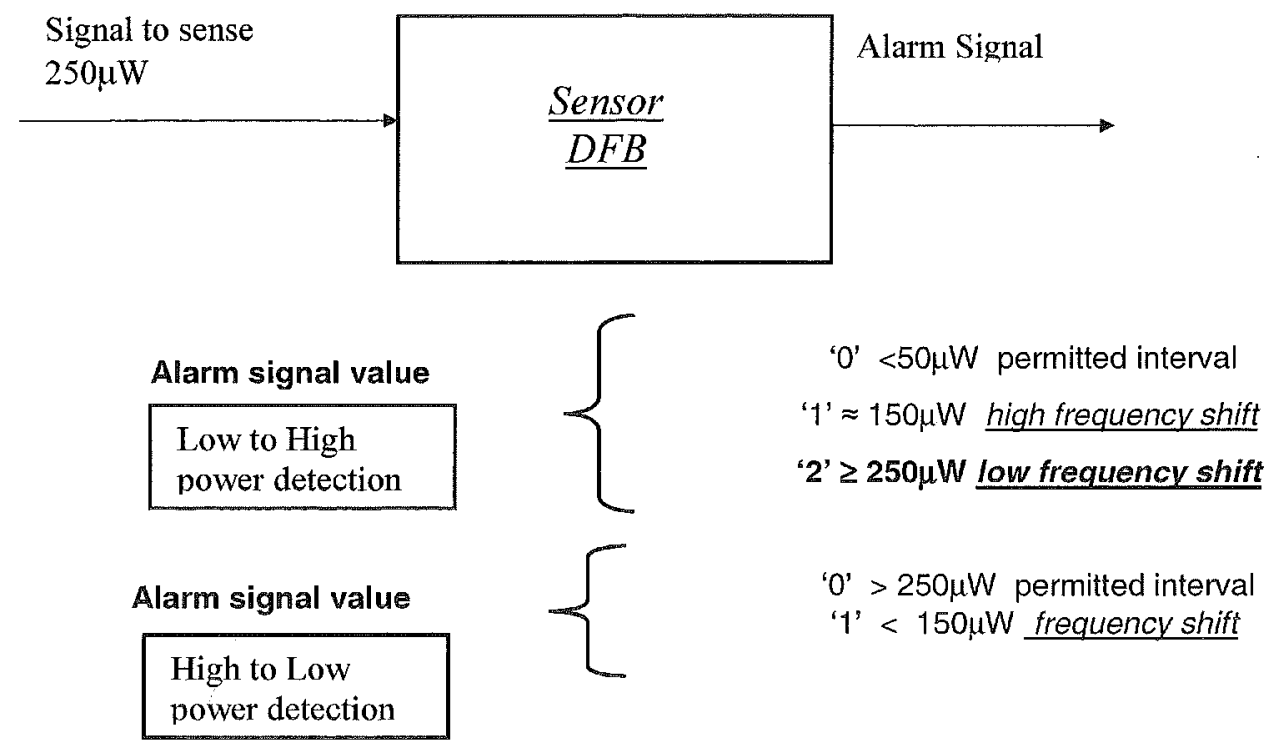

Figure 6.- Summarize of input and output power for frequency detuning. Alarm characterization. Block Sensor is Fig. 1 with DFB laser diode.

reported. We can see that sensor depends on input power. In order to know how much dependence on input power it is reflected on sensor respond on Fig. 7 it is represented the optical output power for different input power on the 
frequency range to evaluate. This dependence gives a freedom on the configuration of the sensor, an attenuator or not

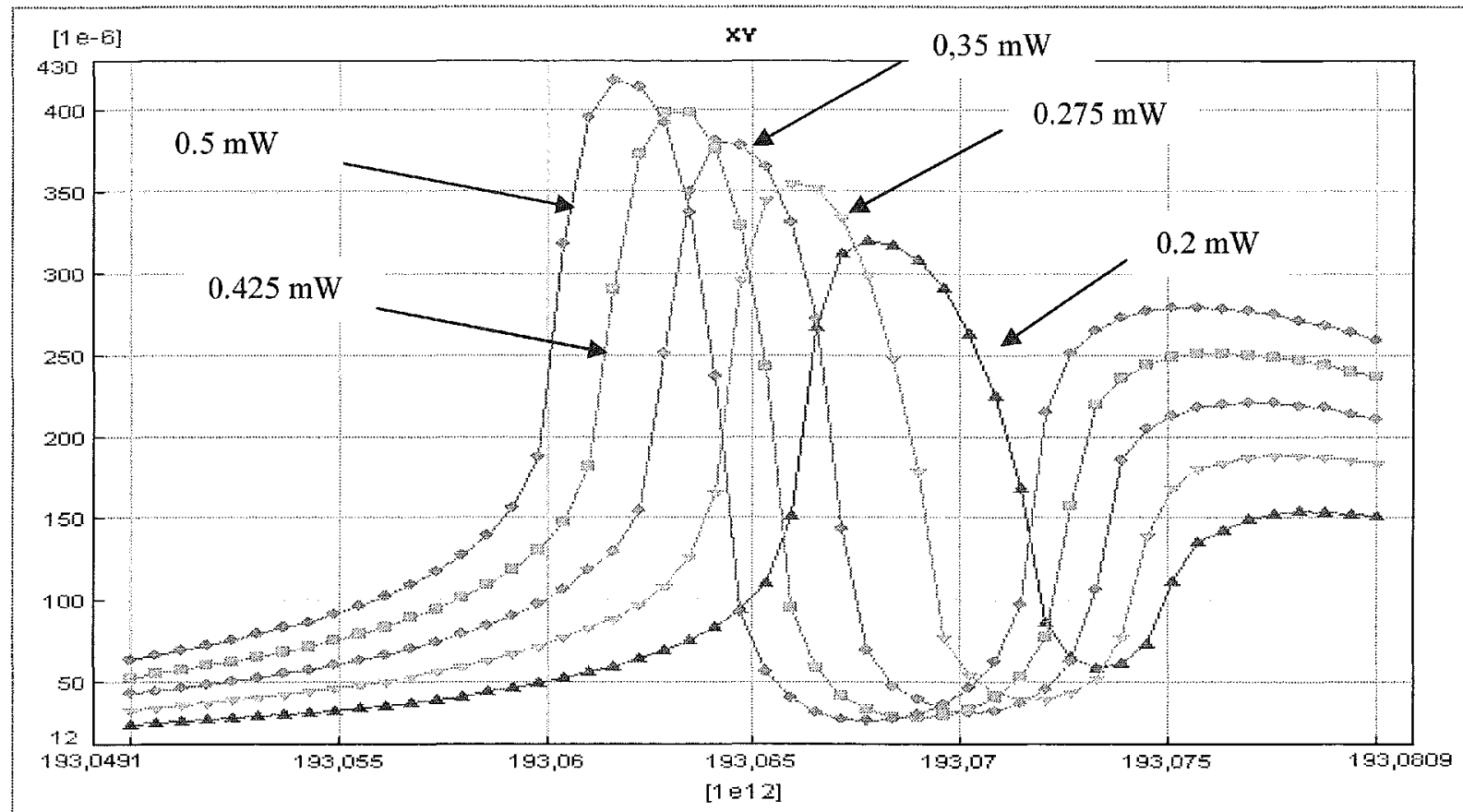

Figure 8.- Semiconductor laser wavelength shift sensor for different values of input power. Range $[0,2 \mathrm{~mW}$ to $0,5 \mathrm{~mW}]$

can be used at the input in order to be sure that the sensor will work on the right bias. A complete characterization depends on the application and fabrication of the laser diode.

Another structure that must be study in order to be sure we can have the cheaper and more suitable sensor is the VCSEL- Vertical Cavity Surface Emitting Laser; even knowing that they are already not totally development on all the desire wavelength range we are studying the bistability behaviour. In this case sensor applications on optical communications may not be very useful, but for optical computing the wavelength it is not that much important or in industrial environment where the wavelength used is not that critical even using optical fibers to transmit signal from

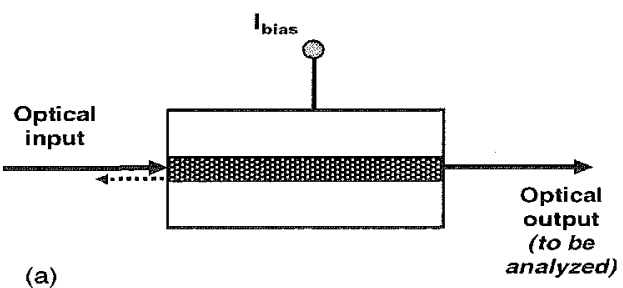

(a)

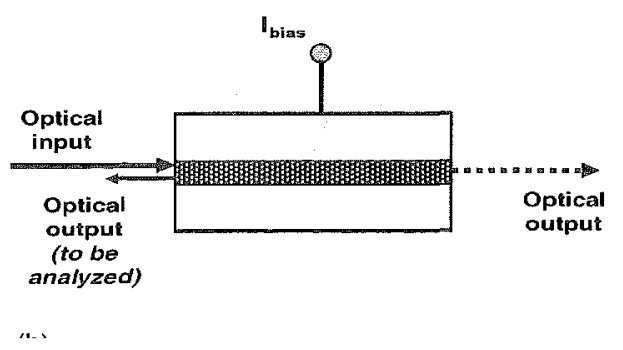

Figure 7.- Basic configurations of the laser diode sensor, (a) by transmission, and (b) by reflection. sensors to process unit or centre. Some result has been reported on [17].

\section{SEMICONDUCTOR LASER AS LIGHT WAVELENGHT SENSOR}

The second configuration to be employed appears in Fig. 8.

The first one (a) corresponds to a classical location, acting the laser diode as diode laser amplifier. The signal to be analyzed impinges to the active region of a laser diode through its frontal surface. Two possible outputs may be studied. The first one (a) is the transmitted light, similar to the obtained from an optical amplifier. A small part will go out from the input surface. The analysis of this light corresponds to the second possible output to be analyzed. This working mode is the reflective mode. In both cases, the characteristics of the output light will be dependent on the relation between the parameters of input light, namely power and optical frequency, and the 
characteristics of the laser diode acting as sensor, bias current and proper optical frequency.

The analysis of the input light characteristics will mainly be based, as it was pointed out before, in the bistable properties of these configurations. The corresponding to the laser diode sensor acting in transmisive mode, that is, taking into account the transmitted light through the rear surface, was reported on [18].

Here we present result for reflection configuration as on Fig.8(b) with a FP laser diode. Curves presented on Figure 9 are the results of output power for different input power on a frequency range. It is show the periodicity of behaviour which is very clear on FP structures. For a given wavelength signal there is a clear range where the output is flat, so we used this to just analyze the output when this go down it means we have the lightwave indicated on the graph.

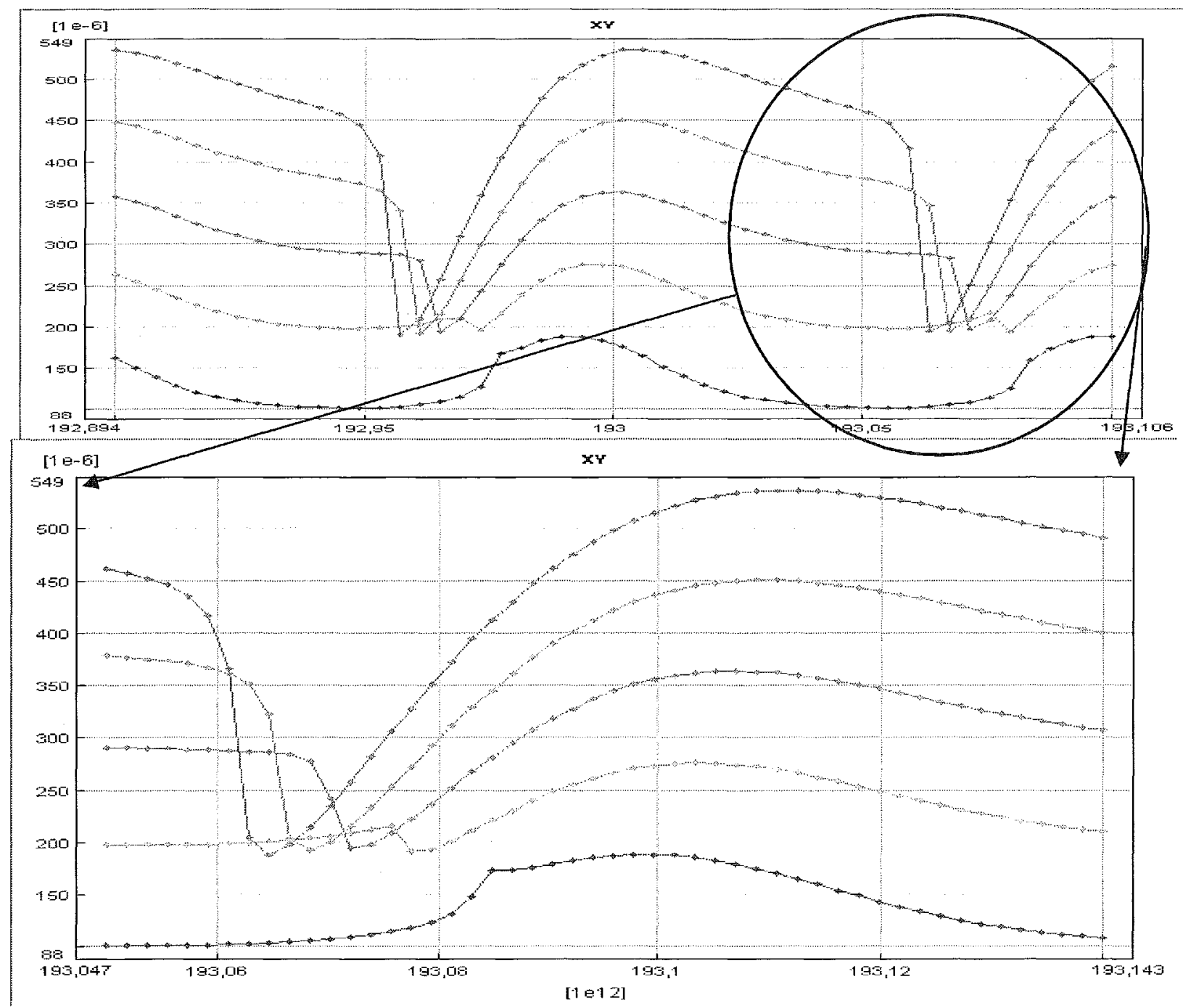

Figure 9.- Frequency (THz) versus optical output Power in $\mu \mathrm{W}$ for different input powers from $0,1-0,5 \mathrm{~mW}$, from structure of Fig. 8 (b) with FP laser diode.

The wavelength for lasing in the sensor is $1552.52 \mathrm{~nm}$. Other parameters for our present laser are depicted in Table I. In all the cases, output intensity is depicted, as customary, as a function of the input intensity. Current crossing the laser diode is, in all the cases, $0.945 \mathrm{I}_{\mathrm{th}}$. As it possible to see in these figures, the change between the two stable states, with lower and higher intensity, is obtained at levels of input power very different, ranging from $500.10^{-6} \mathrm{w}$. This type of sensing in frequency is much better than other previously employed methods. Some more information may be obtained 
from the wideness of the hysteresis cycle, but it needs some more sophisticated methods of measurement. On the contrary, the proposed method allows obtaining the frequency characterization with a very simple instrumentation.

A further parameter of the imput light to be analyzed is its monochromatic characteristics, that is, its line width ${ }^{18}$. Some others, as light polarization, can be studied too.

All the studies done until now has been with the VPI_ComponentMaker ${ }^{T M}$ software tool, the model used by this commercial software it has been reported ${ }^{19}$ and it is well know and validated for our proposes.

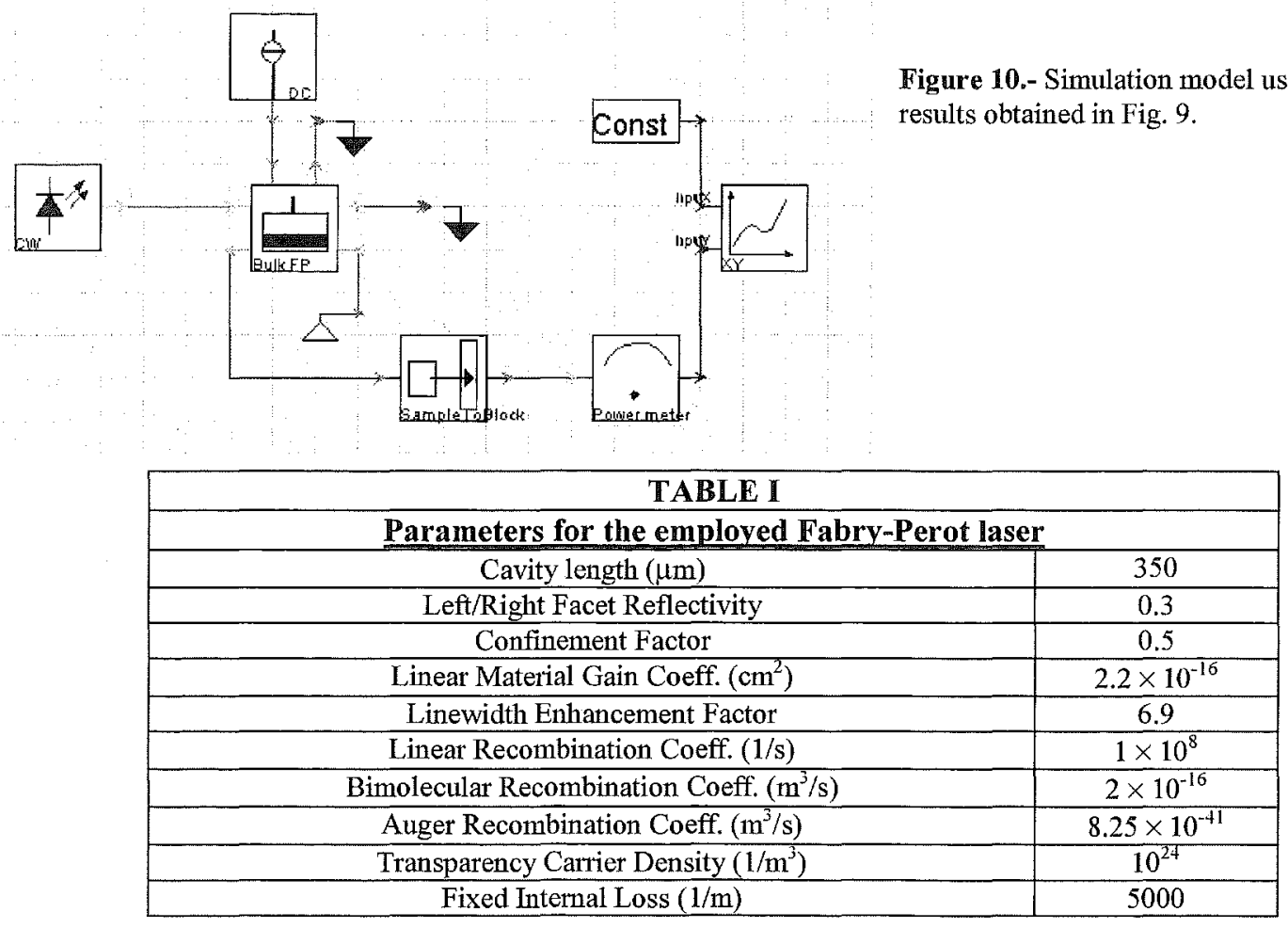

\section{CONCLUSIONS}

Optical logic cells with distributed feedback semiconductor laser diodes DFB-SLD have their potential applications as optical sensors in several functions. Their use allows the implementation of measurement instrument with the same kind of components usually employed in Optical Communications. This implies a very straight forward candidate to a large variety of applications without the need to arrange specific devices.

\section{ACKNOWLEDGMENTS}

This work was partly supported by CICYT, grant TIC2003-04309, and CAM "Comunidad Autónoma de Madrid", grant FPI- Formación de Personal Investigador.

\section{REFERENCES}

1. Lasher, G.J. "Analysis of a proposed bistable injection ${ }^{\circ}$ laser", Solid State Electron., vol. 7 ,pp. 707-716, 1964.

2. Gibbs, H.M. "Optical Bistability: Controlling Light with Light", Academic Press, Inc. 1985.

3. Lattes, A.; Haus, H.A.; Leonberger, F.J. and Ippen, E.P.; "An Ultrafast all-optical gate" IEEE J. of Quantum Electron., vol. QE-19, pp.1718 - 1723, No. 11 Nov. 1983 
4. Otsuka, K.; Kobayashi, S. "Optical bistability and nonlinear resonance in a resonant-type semiconductor laser amplifier" Electro. Lett., Vol. 19, pp.262-263. 1983.

5. Kawaguchi, H.; Inoue, K.; Matsuoka, T.; Otsuka, K. "Bistable output characteristics in semiconductor laser injection locking" IEEE J. of Quantum Electron., vol. 21, pp.1314-1317, no. 9, Sep 1985

6. Shou-Wu, W.; Chiming, W. and Shiming, L. "Stability analysis of semiconductor Bistable lasers" IEEE J. of Quantum Electron., vol. QE-23, pp.1033 - 1038, no.6 June 1987

7. Sharfin, W. F. and Dagenais, M. 'High contrast, 1.3 4 m optical AND gate with gain', in Appl. Phys. Lett. 48, pp. 1510-1512, (1986).

8. Li, L. "Optical frequency bistability and pover bistability in semiconductors lasers" IEEE J. of Quantum Electron., vol. QE-31, pp.233 - 239, No. 2 Nov. 1995

9. Hui, R. "Static and Dynamical Properties of Dispersive Optical Bistability in semiconductor Lasers" J. of Lightwave Technology, vol.13, No.1, January 1995.

10. Miller, D.A.B.; Chemla, D.S.; Damen, T.C.; Wood T.H.; Burrus, Jr C.A.; Gossar, A.C.; Wiegman, W. "The quantum well self-electrooptic effect device : optoelectronic bistability and oscillation, and self-linearized modulation" IEEE J. of Quantum Electron., vol. QE- 21, pp.1462-1476, n.9 , sept.. 1985.

11. Hurtado-Villavieja, A.; Gonzalez-Marcos, A. "An all-optical programmable logic gate with 1550nm laser diode amplifiers", Presented at Optics in Computing 2004, Engelberg (Switzerland), 21-23 April 2004.

12. Hurtado, A.; Gonzalez-Marcos, A. and Martin-Pereda, J.A. "Wavelength monitoring with semiconductor laser amplifiers" Second European Workshop on Optical Fibre Sensors; Jose M. Lopez-Higuera, Brian Culshaw; Eds. Proc. SPIE Vol. 5502, p. 508-511, Jun 2004.

13. Maywar, D.N.; Agrawal, G.P., "Transfer-matrix analysis of optical bistability in DFB semiconductor laser" amplifiers with nomuniform gratings" IEEE J. of Quantum Electron., vol. QE-33, pp.2029 - 2037, no.11 Nov. 1997

14. Maywar, D.N.; Agrawal, G.P., "Effect of chirped gratings on reflective optical bistability in DFB semiconductor" laser amplifiers" IEEE J. of Quantum Electron., vol. 34, pp.2364-2370, no.12, Dec. 1998

15. Hurtado, A.; Gonzalez-Marcos, A. and Martin-Pereda, J.A., "Boolean logic device done with DFB laser diode". Optical Components And Devices. Photonics North, 27 - 29 September 2004 Ottawa (Canada)

16. González-Marcos, Ana P.; Martín-Pereda, José A. and Hurtado, A. "Bit noise from an optical logic gate with laser diodes" SPIE. Fluctuations and Noise in Photonics and Quantum Optics II, vol. 5468, pp. 56-65, (2004)

17. Hurtado, A.; Gonzalez-Marcos, A. and Martin-Pereda, J.A. "Optical Reflective Bistability In Vertical-Cavity Semiconductor Optical Amplifiers (VCSOAs)" Topical Meeting on Optoinformatics, St.Petersburg (Rusia). 18-21 October 2004.

18. González-Marcos, Ana P.; Martín-Pereda, José A. and Hurtado, A. "Laser diode bistability as sensor of optical signal parameters". Fiber Optic Sensors. Photonics North, 27 - 29 September 2004 Ottawa (Canada)

19. Lowery, A.J. "A two-port bilateral model for semiconductor lasers" IEEE J. of Quantum Electron., vol. QE28, pp. $82-92$, n. 1 , Jan. 1992 